\title{
Three-dimensional acousto-optic spectrum analysis
}

\author{
Homayoon Ansari, Brian Metscher, and James R. Lesh \\ Jet Propulsion Laboratory, California Institute of Technology, Mail Stop 161-135, 4800 Oak Grove Drive, Pasadena, California 91109
}

Received March 26, 1990; accepted September 7, 1990

\begin{abstract}
A three-dimensional acousto-optic spectrum analyzer with subhertz resolution is demonstrated experimentally. The first and second dimensions are the two spatial dimensions of the output detector array, and the third dimension is time as sampled by the detector array frame rate. A superfine resolution of $0.12 \mathrm{~Hz}$ has been achieved.
\end{abstract}

In applications involving high-bandwidth search for rf signals, such as the Search for Extra-Terrestrial Intelligence, ${ }^{1}$ it may be desirable to analyze a signal with subhertz resolution once it is identified. Two-dimensional (2D) acousto-optic (A-O) spectrum analysis ${ }^{2}$ has a typical resolution of $100 \mathrm{~Hz}$. The feasibility of superfine ${ }^{2}$ subhertz resolution by extending the operation of a $2 \mathrm{D}$ A-O spectrum analyzer to include time integration over a sequence of output charge-coupled device (CCD) frames and perform three-dimensional (3D) spectrum analysis has been shown analytically. ${ }^{3}$ In this Letter the 3D operation is demonstrated experimentally.

The architecture for a $3 \mathrm{D}$ A-O spectrum analyzer is shown in Fig. 1, and the 3D format at the output CCD array is shown in Fig. 2. A real-time digital image processor (RDIP) is used to grab each $2 \mathrm{D}$ spectral frame that is formed by space and time integration on the CCD array. To obtain each $2 \mathrm{D}$ spectral frame, the signal is first spatially focused in one direction on the CCD array by the Fourier transform (F.T.) lens in Fig. 1 , resulting in coarse resolution. The focused signal is

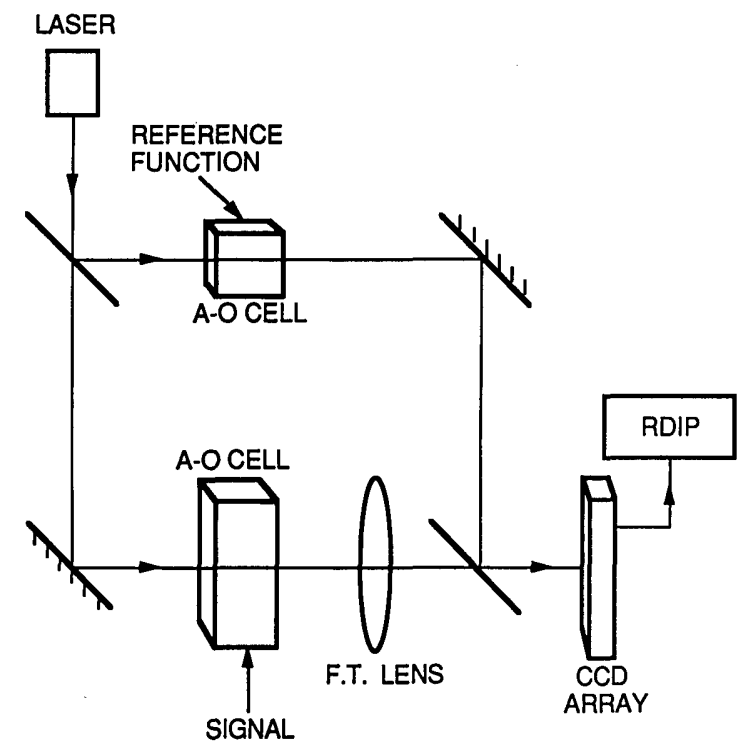

Fig. 1. Three-dimensional A-O spectrum analyzer architecture. then mixed with a reference function in the form of a sampled distributed local oscillator and time integrated on the CCD array, resulting in fine resolution in the orthogonal direction. This is the primary time integration responsible for obtaining a given $2 \mathrm{D}$ spectral frame and identifying a given rf signal with a typical fine resolution of $100 \mathrm{~Hz}$. The two spatial dimensions of the CCD array are thus used to achieve this resolution. A secondary time integration can now be performed at each CCD pixel over a sequence of $2 \mathrm{D}$ spectral frames to obtain subhertz resolution. The third dimension is therefore time as sampled at the CCD array frame rate.

Subhertz resolution is also possible in only one $2 \mathrm{D}$ spectral frame with no secondary time integration. However, for wide analysis bandwidths (e.g., $30 \mathrm{MHz}$ ), this sets stringent requirements on the number of CCD pixels (e.g., 30 million) and the speed of reference function generation. The current $3 \mathrm{D}$ architecture allows for subhertz resolution by using even a commercial, rather than state-of-the-art, CCD array. The 3D operation is particularly suitable for applications in

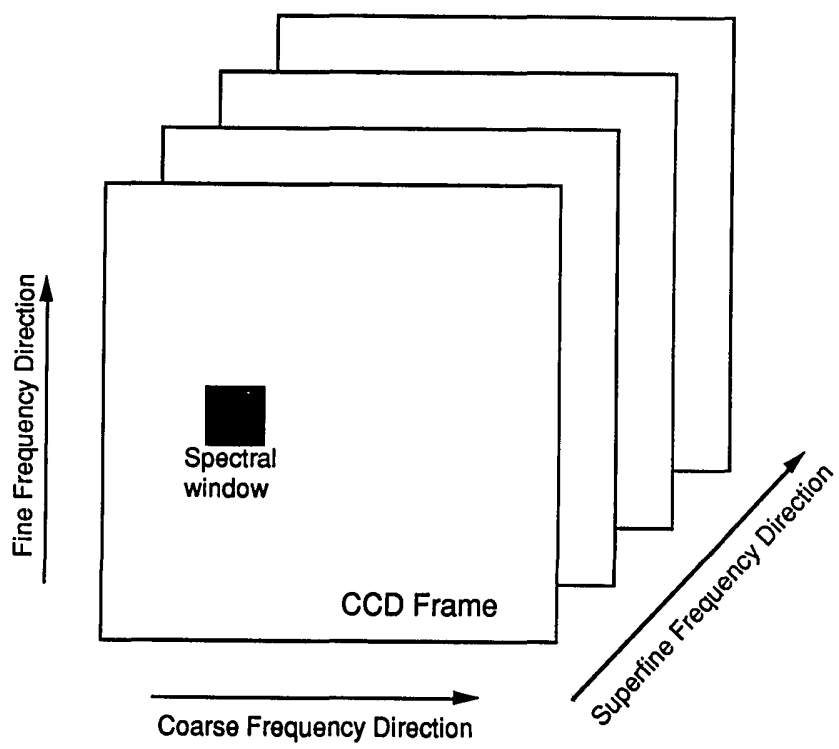

Fig. 2. Coarse, fine, and superfine directions in the 3D A-O spectrum analyzer. 


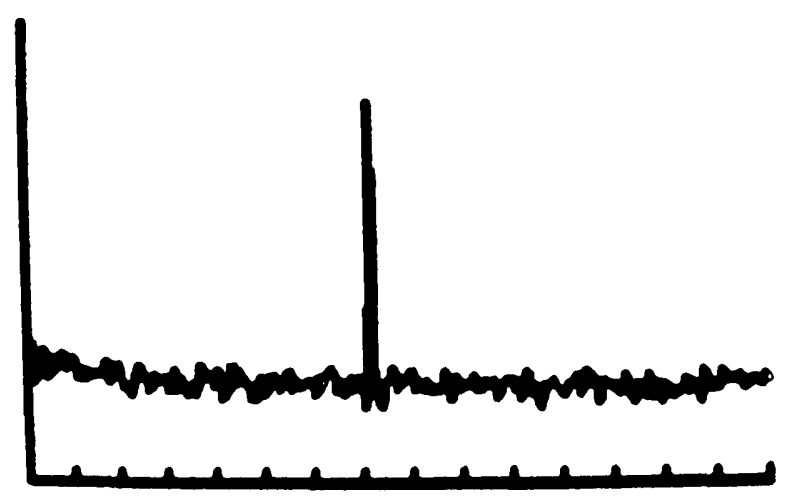

Fig. 3. Third-dimension time-integrated peak for a 1.0009410-MHz input signal. The horizontal scale is $1 \mathrm{~Hz}$ / division.

which a narrow-band $r$ signal composed of a few fineresolution channels is first identified in a wide analysis bandwidth by the 2D operation, and its subhertz features are then to be determined using the third-dimension processing operation.

In the third-dimension time integration, the pixel values in the specified spectral windows of a sequence of CCD frames in Fig. 2 are first read out of the CCD array by the RDIP in Fig. 1. The output of the RDIP is then used by a personal computer to perform time integration on the sequence of values of each pixel in the windows, resulting in a secondarily time-integrat- ed distribution for each pixel. Figure 3 shows the $3 D$ output of the 3D A-O spectrum analyzer for a $1.0009410-\mathrm{MHz}$ input signal. The CCD frame rate was $30 \mathrm{frames} / \mathrm{sec}$, and the secondary time integration was performed over 512 frames or approximately 17 sec. The FWHM of the peak is $0.12 \mathrm{~Hz}$, indicating that a superfine resolution of approximately $0.12 \mathrm{~Hz}$ has been achieved. Two clearly separated peaks were observed when using two signal components with a $0.2-\mathrm{Hz}$ separation (the frequency increment of the input signal generator was $0.1 \mathrm{~Hz}$, and therefore a frequency separation of exactly $0.12 \mathrm{~Hz}$ could not be tested).

In conclusion, 3D operation and subhertz resolution in an A-O spectrum analyzer have been experimentally demonstrated by third-dimension time integration using a sequence of $2 \mathrm{D}$ spectral frames.

This research was carried out at the Jet Propulsion Laboratory, California Institute of Technology, under contract with the National Aeronautics and Space Administration.

\section{References}

1. M. J. Klein, S. Gulkis, E. T. Olson, and N. A. Renzetti, Jet Propulsion Laboratory TDA Progress Report 42-98 (California Institute of Technology, Pasadena, Calif., 1989), p. 218.

2. K. Wagner and D. Psaltis, Proc. Soc. Photo-Opt. Instrum. Eng. 564, 209 (1985).

3. H. Ansari and J. Lesh, "Superfine-resolution acoustooptic spectrum analysis," submitted to Appl. Opt. 